\title{
Development of a Low-Cost Quadrupedal Starfish Soft Robot
}

\author{
M. Munadi \\ Mechanical Engineering \\ Department \\ Diponegoro Univeristy \\ Semarang, Indonesia \\ muna 096@yahoo.com
}

\author{
Mochammad Ariyanto \\ Mechanical Engineering \\ Department \\ Diponegoro Univeristy \\ Semarang, Indonesia \\ arijanto5@yahoo.co.id
}

\author{
Joga D. Setiawan \\ Mechanical Engineering \\ Department \\ Diponegoro Univeristy \\ Semarang, Indonesia \\ joga.setiawan@gmail.com
}

\author{
M. Fikri Al Ayubi \\ Mechanical Engineering \\ Department \\ Diponegoro Univeristy \\ Semarang, Indonesia \\ ayubi.fikri@gmail.com
}

\begin{abstract}
In this paper, a low-cost soft quadrupedal starfish soft robot will be developed. The proposed soft robot's body has a shape with $X$ configuration. The soft legs of the robot's material utilizes silicon rubber that has been available on the market especially in online store. While, poly lactic acid (PLA) is used as the material of robot's body. The soft leg of the robot employs tendon-based mechanism. Micro servomotors are selected for the actuation system. The electrical components must be lightweight enough in order to the robot can carry the load. Undulating gait was developed and implemented on the soft robots to move the soft legs in sequence and create a wave of motion that moves the entire robot body. The gait cycle equation is obtained using Fourier series. Based on the test result, the proposed starfish soft robot can walks in straightforward direction using undulating gait cycle
\end{abstract}

Keywords-low-cost, quadrupedal, starfish, soft robot

\section{INTRODUCTION}

Robot technology is growing rapidly, including materials or materials for making robots. Most robots use rigid materials such as iron, aluminum, plastic and so on. When illustrating robots that resemble biological creatures, rigid components are very difficult to implement. The constituent materials used for the robot have at least soft, elastic and flexible properties [1]. It aims to enable robots to survive in complex and unpredictable environmental conditions. An example is an animal, its body part consists of soft skin, hair, elastic tendon muscles and various organs of the body all of which have soft texture.

Recently, the development of soft robot increased rapidly. This is due to a better understanding of mechanical systems, and the incorporation of biological fields. In the development of soft robot, there is still some obstacle, including in term of speed. Most soft robots are developed with a pneumatic actuator mechanism, which for soft robots that can run using this type of actuator is less suitable because of its slow movement.

Soft robot is inspired by how living organisms move and adapt to their environment. In contrast to the hard robots made using rigid materials, soft robot allows the addition of flexibility and the ability to adapt its working environment in order to perform its tasks, as well as improve work safety [2].

Besides the robot design must be as light as possible, so that the actuation system work is not too heavy and the robot does not consume excessive power. In order to robot can survive in all conditions, the robot must be made of a reliable material. One of the criteria, the robot must be flexible so that when an impact occurs, the robot does not experience serious mechanical problems. In this study, the robot uses tendon-based mechanism as the actuation system. Previous research employs pneumatic network actuation such as in ref. [3-5]. The pneumatic actuation implemented on the soft robot has good performance in the previous research results. Other starfish soft robots employs shape memory alloy (SMA) wires such as [6-8]. The locomotion is inspired by starfishes. The proposed starfish robots can crawl and walk using predefined locomotion.

In this research, quadrupedal starfish soft robot is designed to carry all of the electrical components on the body. The body design must be as efficient as possible, so as not to leave too much space that can be utilized or lack of space so that electronic components cannot be minimized compactly. The legged locomotion of the robot is derived from Fourier series equation.

\section{QUADRUPEDAL STARFISH SOFT ROBOT DESIGN AND MANUFACTURE}

In the design stage, some quadrupedal soft robot configurations are studied. This is conducted to find the best configuration design so that the quadrupedal soft robot is able to carry out the research mission as well as possible. In this study, the mission of the quadrupedal soft robot is able to move in different directions. To fulfill this mission soft robot must be able to fulfill several important points as follows:

- Soft robot has a shape with X configuration

- The body design of quadrupedal starfish soft robot should be compact so it has a minimalist and slim shape

- Quadrupedal starfish soft robot must be able to walk forward, backward, turn left and right

- Quadrupedal starfish soft robots must be able to be controlled wirelessly at a certain distance

- Quadrupedal starfish soft robots should be able to transmit data and images in real time

\section{A. Soft Actuator Design}

From several comparisons of actuators that are widely used in soft robot, tendon based actuator is selected as actuation system for quadrupedal starfish soft robot. In term of durability, this robot can withstand extreme conditions without worrying about leakage in the actuators such as actuators that use pneumatic networks. This tendon type 
actuator does not require expensive costs in the manufacturing process.

The design developed in this study is one of the untethered systems that combine a set of functional elements (body, power supply system, control algorithm, etc.). This robot is designed as a starting point for the development of untethered soft robot using a tendon based mechanism. In the design of the molding actuator, there are several things to consider. The first is the functionality aspect of the actuator and the next is the ease of performing a series of casting process. In carrying out this series of studies, the actuator and the mold were made several times.

In the soft leg robot actuator design, there are several things that are improved from the previous design. Among them are fillers made at the same time as joints, so that they become the right joint between the body and the actuator. In addition, the use of hot glue is not used and replaced by wax. The wax serves as a seal so that the molding does not leak. After casting, the final molding design is the most effective actuator and mold design, with the reason that the casting process is relatively easy, and does not require additional tools such as hot glue, cutter and so on. The casting results for quadrupedal starfish soft robot legs are as expected. The design for soft robot actuator in computer aided design (CAD) and the resulted prototype can be seen in Fig. 1.

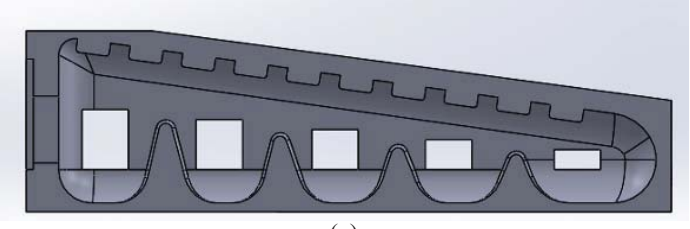

(a)

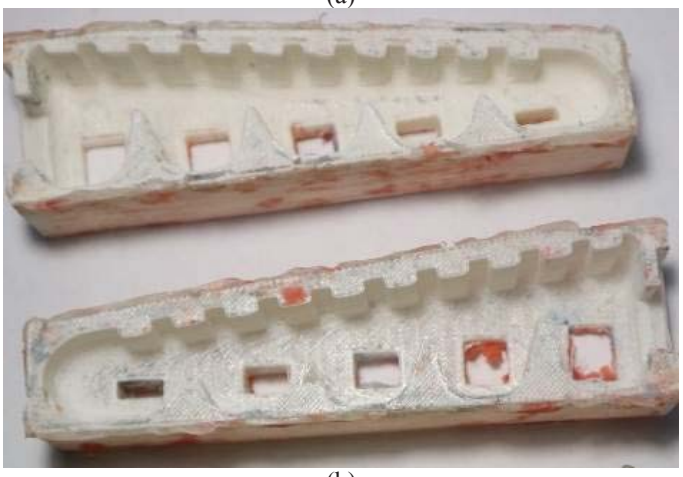

(b)

Fig. 1. 3D printing of mold, (a) CAD design (b) Resulted mold

\section{B. Body Design}

In the developing body design, several design changes were made. The changes such as less efficient design, the body still too heavy, lack of space due to planning errors and so forth. In the latest body design, most of the problems on the previous designs have been resolved. With the addition of vents throughout the robot, it is expected to reduce the weight of the robot. The ventilation also functions as a place for air flow which aims to cool the components of the actuator components of the robot.

In the final design, the body is equipped with switch socket and LED, making it easier to access the robot. In this body design also has a lighter weight, because there is a lot of ventilation/hollow. Body thickness is also reduced to a size of only $3 \mathrm{~mm}$, which was previously made with a thickness of $5 \mathrm{~mm}$. The final body design and the printed body is shown Fig. 2 and Fig. 3.
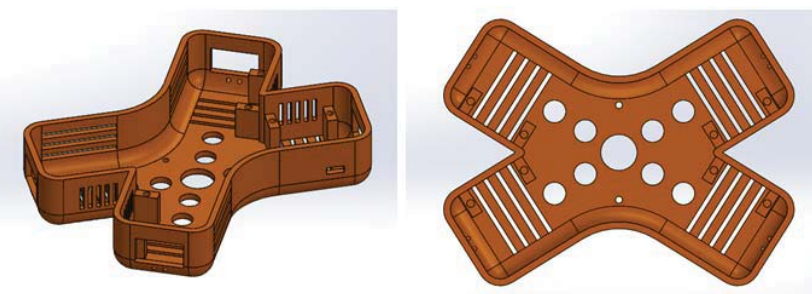

Fig. 2. CAD design of soft robot's body

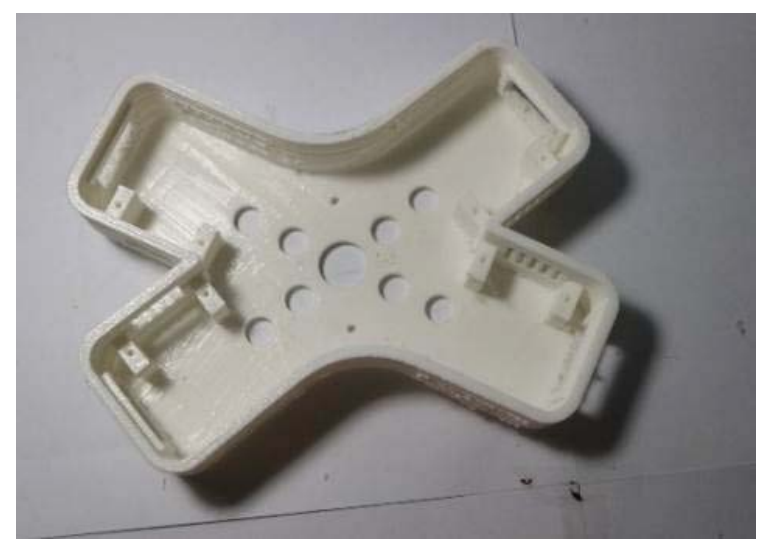

Fig. 3. 3D print body of quadrupedal soft robot

\section{Soft Leg Manufacture}

The material used as the actuator is synthetic rubber. Topco KCT 291 was chosen because it has the appropriate rigidity based on the robot need, and also based on the availability of actuators in the market. Topco KCT 291 stiffness point is between Room-Temperature-Vulcanizing silicone (RTV) 48 which is too low in stiffness so that the actuator using this material cannot work properly and RTV platinum has twice the rigidity of Topco KCT 291. So that the drive is needed which has a large torque that affects the dimensions of the robot.

In addition when casting, Topco KCT 291 can fill a narrow gap so that the actuator can be formed perfectly, as well as other RTVs. But the concern here is the result of the casting, where RTV 48 produces the final form of the actuator that is not in accordance as the expected, such as the number of fiber molding that is printed so that the casting surface becomes less attractive. Topco KCT 291 is a silicone rubber that has low viscosity and has a transparent color. Silicone rubber has a curing time is fast, long pot life and have a good mechanical strength. This material consists of methyl vinyl silicone polymer which has a high purity.

After all the molding preparations are performed as shown in Fig. 4, then then molding material consisting of silicone rubber is prepared. The first step in making dough is to weigh silicone rubber of 90 grams, give a special dye of 1 gram on the silicone, and mix it. After the dyes and silicone are mixed, a catalyst is added to the mixture as much as 1 gram. The ratio between silicone and catalyst is 100: 1. After the dyes and catalysts are well blended, the dough is poured into the mold as shown in Fig. 5. 


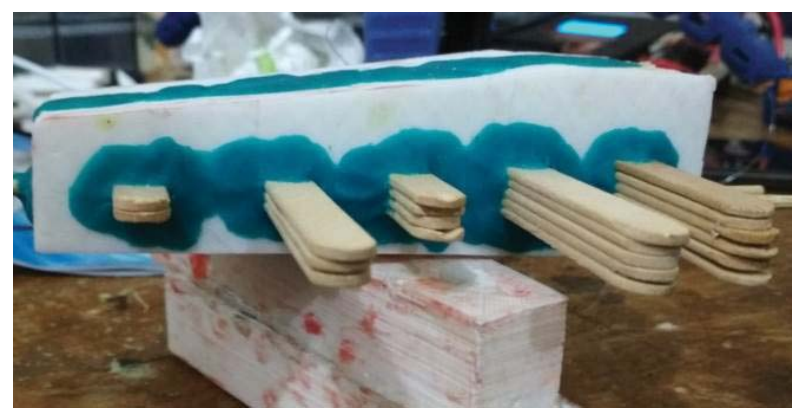

Fig. 4. Complete soft actuator mold

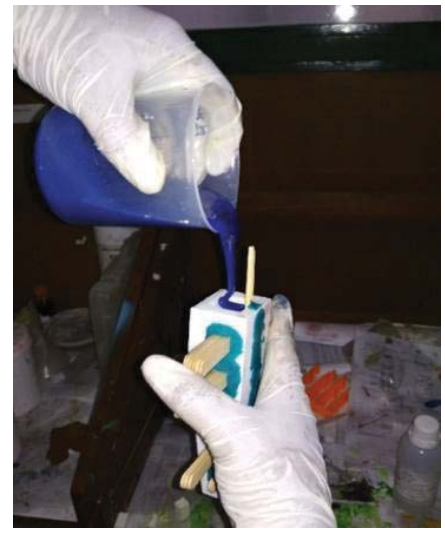

Fig. 5. Pouring the silicone mix to the mold

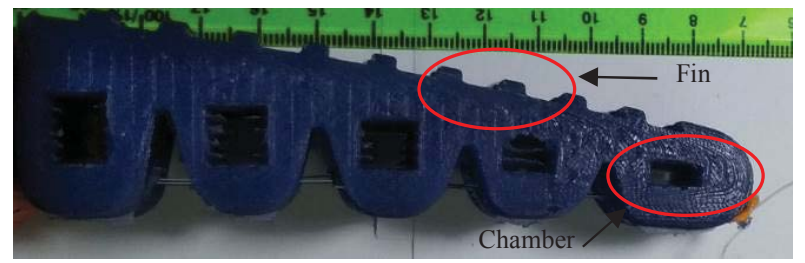

Fig. 6. Soft leg actuator

\section{QUADRUPEDAL STARFISH SOFT ROBOT SYSTEM}

At the beginning of the study, the quadrupedal starfish soft robot actuator was driven by using TowerPro MG90s servo, but after assembly along with the other parts, the servo could not pull the actuator using the tendon properly. The servo motor was later changed to Turnigy TGY-9018MG, which theoretically has a slight torque beneath the MG90s, but it has better quality.

Because the aim of developing a quadrupedal starfish soft robot is to get an actuator component and a microcontroller circuit that can be installed and attached inside the body, then a small microcontroller is needed but still able to execute complex commands. Arduino Nano is one variants of Arduino board microcontroller products. The Arduino Nano is the smallest Arduino board. The board uses Atmega 328 microcontroller.

NRF24L01 trans-receiver module uses wireless communication with $2.4 \mathrm{GHz}$ bandwidth width and can be operated on baud rate from $250 \mathrm{Kbps}$ up to $2 \mathrm{Mbps}$. When used in open spaces with low baud rate values, the distance that can cover 100meter. Based on these considerations, NRF24L01 was selected in this study.

Joystick module was used to provide remote commands. This module has five pins: VCC, Ground, X, Y, and Key. The order in each joystick module will vary depending on the module manufacturing plant. Analog (thumb stick) will provide more accurate readings than simple directional joystick using push buttons or using mechanical switches. The joystick can be pressed to gain additional functionality.

In this study, four-servo motors were used for pulling the soft leg actuator as shown in Fig. 6. The tip of the soft leg is connected from servo motor using braided string. The electrical components must be lightweight enough in order to the robot can carry another payload. The schematics of the utilized electrical components on the robot body and the remote control are presented in Fig. 7 and Fig. 8. The final prototype of the proposed soft quadrupedal soft robot is shown in Fig. 9.

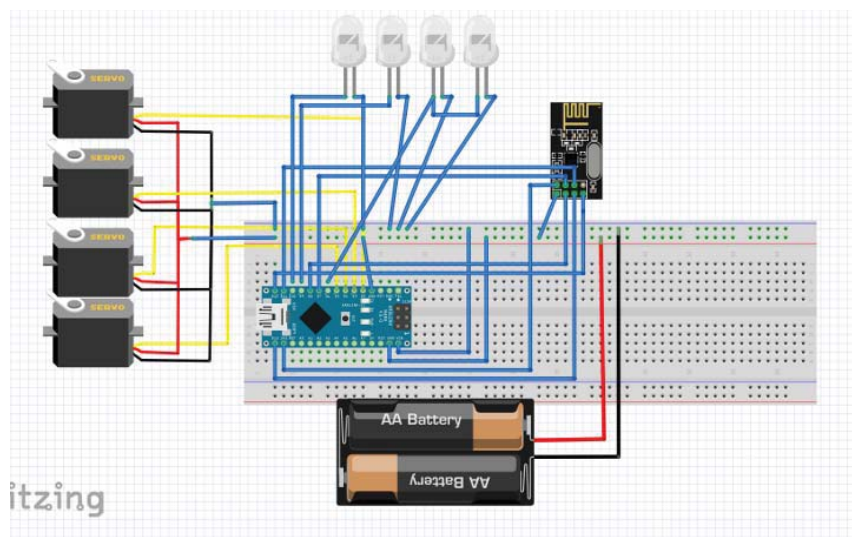

Fig. 7. Schematic of electrical components on the robot body.

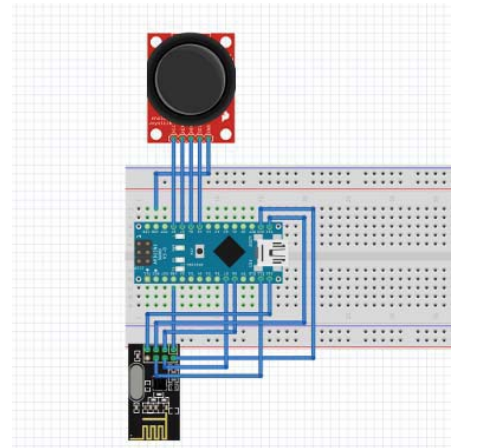

Fig. 8. Schematic of electrical components on the remote control

The prototype of quadrupedal starfish soft robot that has been developed can walk using four soft actuators with an undulating gait pattern. In this study, undulating gait was implemented on the soft robots to move the soft legs in sequence and create a wave of motion that moves the entire robot body from behind to the front. This condition produces forward movement. The undulating gait system consists of five conditions that run sequentially and repeatedly as follows:

- Two rear legs move together

- The rear legs, and the body then moves together

- The rear legs, body and front legs then move together 
- The front legs move while the body and rear legs do not, the time difference in the movement between the front legs and the rear legs causes the body to lean forward

- All actuators turn off to allow the servo time to rest and conserve battery power

- The front left and front right legs have the same movement, while the rear left and rear right legs have the same gait motion as shown in in Fig. 10.

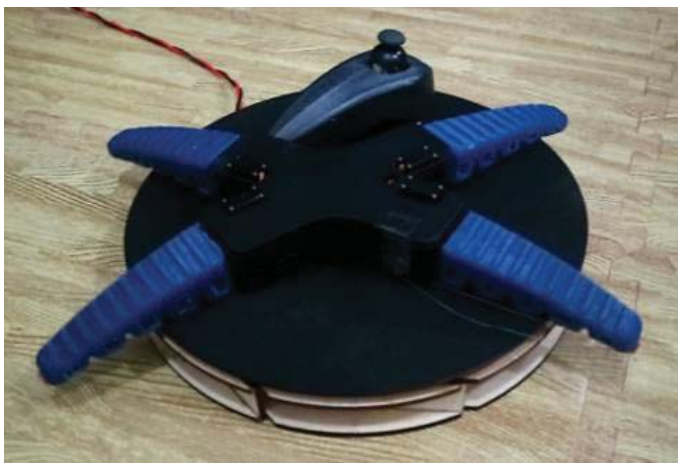

Fig. 9. Final prototype of the soft quadrupedal starfis robot

The designed undulating gait pattern for each leg as shown in Fig. 10 is processed by using curve fitting with Fourier series. The acquired Fourier series equation can expressed as in equation (1). Where $f$ is the amplitude of the undulating gait, $t$ is time and $\omega$ is the bending frequency of the soft legs in $\mathrm{rad} / \mathrm{s}$.

$$
\begin{gathered}
f(\mathrm{t}, \omega)=\mathrm{a}_{0}+\mathrm{a}_{1} \cos (\omega t)+\mathrm{b}_{1} \sin (\omega t) \\
\ldots .+\mathrm{a}_{2} \cos (2 \omega t)+\mathrm{b}_{2} \sin (2 \omega t) \\
\ldots .+\mathrm{a}_{3} \cos (3 \omega t)+\mathrm{b}_{3} \sin (3 \omega t) \\
\text { Undulating gait cycle }
\end{gathered}
$$

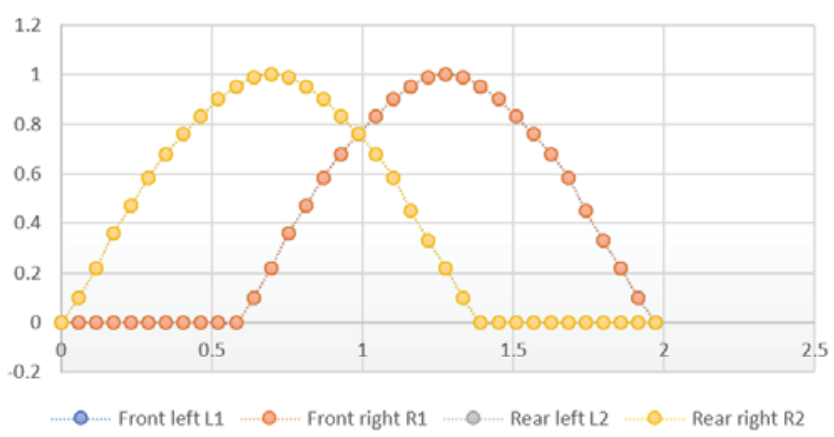

Fig. 10. Undulating gait design

\section{RESUlt AND Discussion}

By using curve fitting with Fourier series, the gait cycle equation is obtained using the Fourier series equation. After Fourier's mathematical equations were obtained, the undulating gait mathematical equation (1) was embedded into Arduino Nano V3.0. Undulating gait as shown in Fig. 11 shows the visualization of motion generated by the gait using the Fourier equation and implemented into the robot starfish system. From gait cycle as shown in Fig. 11, it can be seen that the robot can do one-time gait cycle by taking two seconds to complete. One-step undulating gait on the robot can be shown in Fig. 12. The Figure shows that the front legs and rear legs move in sequence.

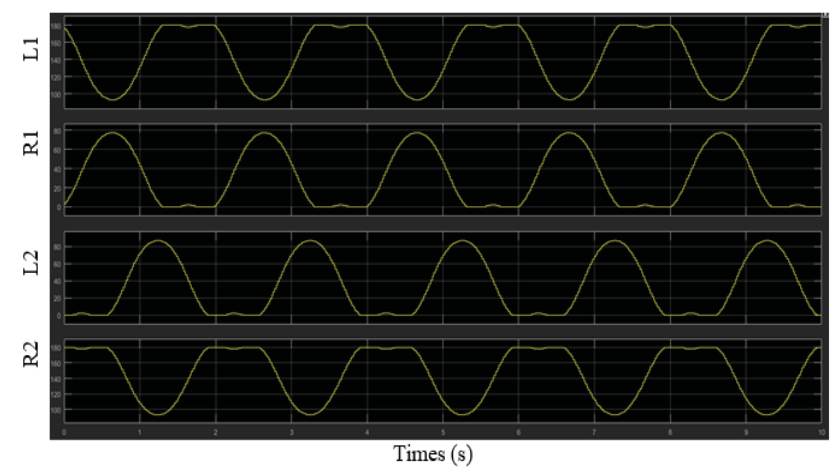

Fig. 11. Gait command for soft legs embedded into the Arduino

The force on each soft actuator on the robot affects the soft tendon ability of the actuator in carrying the load. In a simple test, the soft robot carries a load that has previously been determined from a state of full relaxation to a state of full contraction. As shown in Fig. 13, where the maximum power of this robot can carry the maximum load is 350 grams. This is affected by the torque limitations of the servo motor which has only $1.8 \mathrm{~kg} . \mathrm{cm}$ of torque each servo motor.

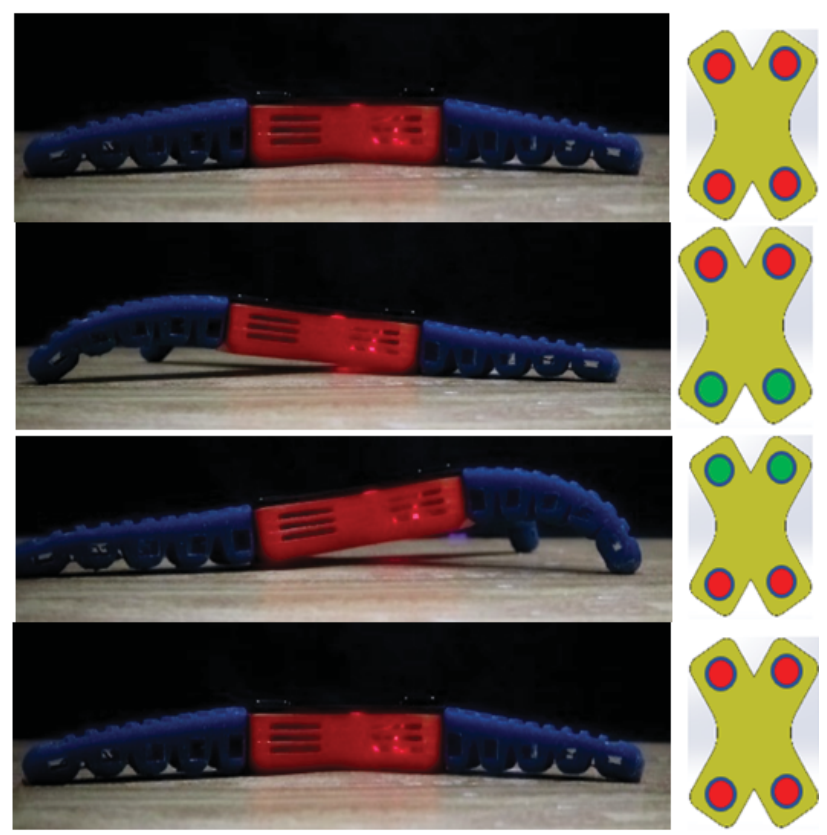

Fig. 12. Undulating gait with one cycle step

\section{CONCLUSION}

In this study, quadrupedal starfish robot has been successfully developed with a tendon based mechanism using Topic KIC 291 silicone rubber material for soft legs and Polylactic Acid (PLA) for the body of robot robot. Dimensions of the resulting robot is $\mathrm{p} \mathrm{x} 1 \mathrm{x}$ t: $350 \mathrm{~mm} \times 230$ $\mathrm{mm} \times 32 \mathrm{~mm}$ with a total weight of 650 grams. The starfish robot can walks in straightforward direction using undulating gait cycle. The sequnce images of the soft starfish robot walks using the undulating gait is depicted in Fig. 14. The Fourier serier can be succesfully implemented for legged locomotion of the robot for walking. 


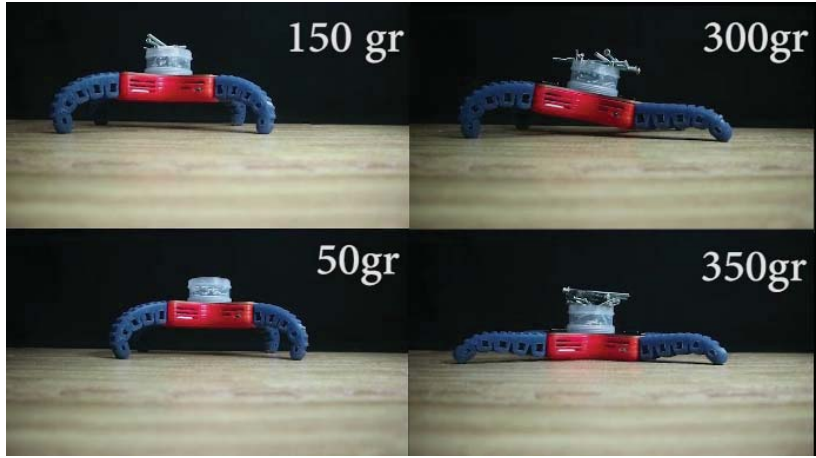

Fig. 13. Payload lift test

The manufacturing process for soft actuator quadrupedal starfish should use a vaccum chamber and silicone rubber with better quality (elongation). This is because the cavity and flexibility of each actuator is not the same in this study because it was made on a different days. In the future research, the control algorithm for turn left, turn right will be developed using Fourier series. Another gait pattern will be studied such as ambulating gait. Obstacle avoidance also will be incorporating in the gait walking of the robot. The actuator and body design can be made more flexible and lighter to improve the quadrupedal starfish soft robot performance.

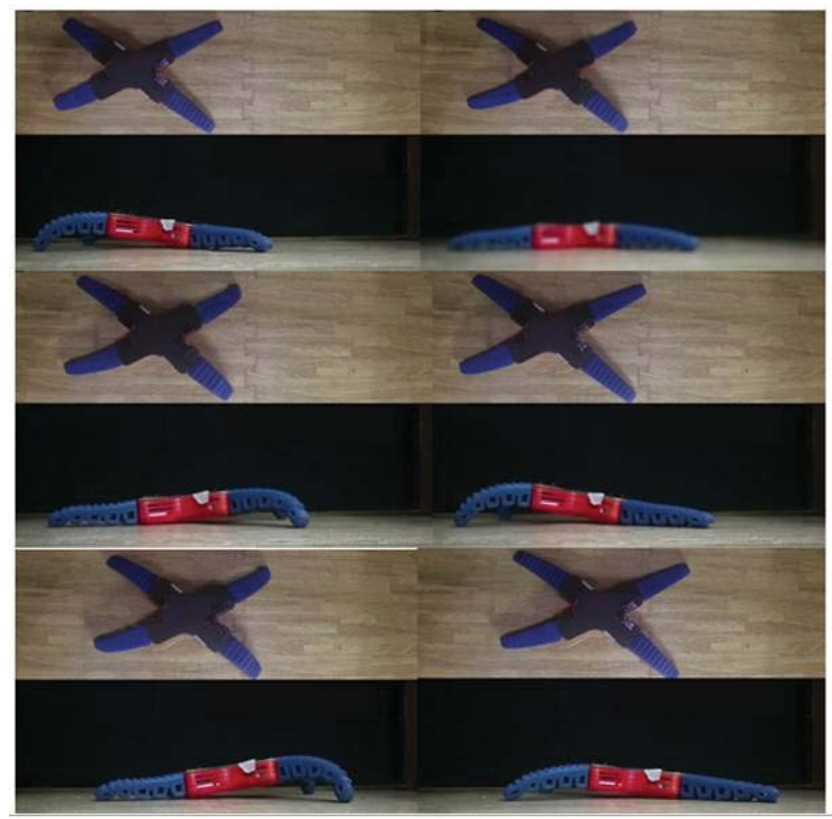

Fig. 14. Starfish soft robot walks straighforward

\section{Acknowledgment}

This work is supported by Diponegoro university research fund under International Publication Research scheme.

\section{REFERENCES}

[1] Iida, Fumiya, and Cecilia Laschi. "Soft robotics: challenges and perspectives." Procedia Computer Science 7 (2011): 99-102.

[2] Tolley, M. T. \& Rus, D., 2015. Design, fabrication and control of soft robots. Nature, 521(7553), p. 467-475.

[3] R. F. Shepherd et al., "Multigait soft robot," Proc. Natl. Acad. Sci., vol. 108, no. 51, pp. 20400-20403, Dec. 2011.

[4] A. Poungrat and T. Maneewarn, "A starfish inspired robot with multidirectional tube feet locomotion," 2017 IEEE International Conference on Robotics and Biomimetics (ROBIO), Macau, 2017, pp. 712-717.

[5] D. Drotman, S. Jadhav, M. Karimi, P. deZonia and M. T. Tolley, "3D printed soft actuators for a legged robot capable of navigating unstructured terrain," 2017 IEEE International Conference on Robotics and Automation (ICRA), Singapore, 2017, pp. 5532-5538.

[6] Mao, S., Dong, E., Jin, H., Xu, M., Zhang, S., Yang, J., \& Low, K. H. (2014). Gait Study and Pattern Generation of a Starfish-Like Soft Robot with Flexible Rays Actuated by SMAs. Journal of Bionic Engineering, 11(3), 400-411.

[7] H. Jin, E. Dong, G. Alici, S. Mao, X. Min, C. Liu, K. H. Low, and J. Yang, "A starfish robot based on soft and smart modular structure (SMS) actuated by SMA wires," Bioinspiration \& Biomimetics, vol. 11, no. 5, p. 056012, Sep. 2016.

[8] S. Mao, E. Dong, M. Xu, H. Jin, F. Li and J. Yang, "Design and development of starfish-like robot: Soft bionic platform with multimotion using SMA actuators," 2013 IEEE International Conference on Robotics and Biomimetics (ROBIO), Shenzhen, 2013. 